\title{
Triterpene-loaded microemulsion using Coix lacryma-jobi seed extract as oil phase for enhanced antitumor efficacy: preparation and in vivo evaluation
}

\author{
This article was published in the following Dove Press journal: \\ International Journal of Nanomedicine \\ 19 December 2013 \\ Number of times this article has been viewed
}

\section{Ding Qu \\ Junjie He \\ Congyan Liu \\ Jing Zhou \\ Yan Chen}

Key Laboratory of New Drug Delivery System of Chinese Materia Medica, Jiangsu Provincial Academy of Chinese Medicine, Jiangsu, People's Republic of China
Correspondence: Yan Chen Key Laboratory of New Drug Delivery System of Chinese Materia Medica, Jiangsu Provincial Academy of Chinese Medicine, 100 Shizi Road, Nanjing, Jiangsu 210028, People's Republic of China Tel +862585608672

Fax +862585637809

Email ychen202@hotmail.com

\begin{abstract}
Ganoderma lucidum triterpene-loaded microemulsions (TMEs) using Coix lacrymajobi (adlay) seed oil as oil phase were prepared, characterized, and evaluated for enhanced antitumor activity. Ternary phase diagrams for the TMEs were constructed and the optimal preparation was developed. Transmission electron microscopy and dynamic light scattering showed that this formulation had a well defined spherical shape, a homogeneous distribution, a small size, and a narrow polydispersity index. The drug-loading rate was determined to be $9.87 \%$ by ultraviolet spectrophotometry, and acceptable stability under various stimulations in vitro was confirmed. Importantly, the TME formulation showed a significantly greater antiproliferative effect towards human lung carcinoma (A549) cells and murine lung tumor (Lewis) cells in comparison with suspension formulations containing triterpene and adlay seed oil as a positive control. The half-maximal inhibitory concentration of the TMEs was about $0.62 \mathrm{mg}$ crude drug per $\mathrm{mL}$, being 2.5 -fold improved relative to that of the corresponding suspension formulation, but no significant cytotoxicity was observed for the bare microemulsion in A549 cells and Lewis cells. In vivo, the TME formulation showed markedly enhanced antitumor efficacy in a xenograft model of Lewis lung cancer after intragastric administration. Compared with cyclophosphamide, the TME formulation showed similar antitumor activity but less general toxicity. These results indicate the feasibility of using a microemulsion to increase the solubility of triterpene and adlay. TMEs hold promise as an efficient drug delivery system for the treatment of lung cancer.
\end{abstract}

Keywords: microemulsion, Ganoderma lucidum, triterpene, adlay seed oil, lung cancer

\section{Introduction}

Ganoderma lucidum ("Ling-Zhi" in the Chinese language) is a plant with widely recognized health benefits and has been used extensively in the People's Republic of China and other eastern countries for over 2,000 years. The major bioactive ingredients in G. lucidum are polysaccharides, triterpene, and adenosine, which have antitumor and immunomodulatory activity. ${ }^{1-4}$ Meanwhile, Coix lacryma-jobi (adlay), used as a nourishing food in traditional Chinese medicine, has proven activity in reducing the risk of cancer. ${ }^{5}$ In recent years, an adlay seed oil emulsion has been approved for use as an antineoplastic therapy by the Chinese Ministry of Public Health, in particular for lung cancer. In addition, a Phase I clinical trial of adlay seed oil has been done in Russia, and evaluation of adlay extract for common types of cancer therapy has also been done in the US. ${ }^{6}$ 
According to traditional Chinese medicine theory, coadministration of G. lucidum and adlay has synergistic effects such as improving qi, nourishing yin, eliminating dampness (diuretic effects), inhibiting tumors, and resolving hard lumps. Based on the confirmed antitumor activity of the above natural products, we have previously used a formulation containing G. lucidum and adlay, known as "Ling-Yi", and found it to have clear antitumor activity in lung cancer. In view of its complex components, most research groups have explored the antitumor effect of G. lucidum by using specific extracts of the plant, eg, triterpene, which has demonstrated cytotoxicity against cancer cells, in preference to total extracts, which may have a more comprehensive but weaker physiologic effect. ${ }^{7-10}$ Likewise, adlay seed oil, extracted from adlay using supercritical $\mathrm{CO}_{2}$, has been used in the treatment of cancer for a number of years. However, due to the particularly low solubility and poor permeability of triterpene and adlay seed oil, the Ling-Yi formulation still presents several challenges because of its unacceptable biopharmaceutical performance.

Nowadays, nanotechnology, which includes microemulsions, liposomes, nanoparticles, and polymeric micelles, is becoming an increasing focus for a large number of cancer researchers throughout the world. ${ }^{11-14}$ For instance, microemulsions, commonly prepared by surfactant, cosurfactant, oil, and drug, are thermodynamically stable and transparent or translucent colloidal dispersions with a small size and low viscosity. ${ }^{15-17}$ In recent years, microemulsions have become an efficient approach to improving the solubility and poor oral bioavailability of insoluble drugs. ${ }^{18-20}$ Given the relative low intestinal absorption of triterpene and adlay seed oil, introduction of microemulsion technology into the Ling-Yi formulation might be helpful in overcoming its intrinsic disadvantages, improving its bioavailability, and enhancing its therapeutic effect. However, a microemulsion containing a high concentration of a pharmaceutical adjuvant could potentially have a detrimental effect on normal body tissue. ${ }^{21,22}$ A comprehensive survey of traditional Chinese medicines indicates that various components, such as saponin and volatile oil, enable effective replacement of the surfactant and oil phase to reduce unnecessary adjuvant content and improve safety. ${ }^{23,24}$

In this study, a triterpene-loaded microemulsion (TME) formulation containing adlay seed oil as oil phase was developed and characterized. The main purpose of the study was to compare the in vitro and in vivo antitumor efficacy of the TME and suspension formulations of triterpene and adlay seed oil, identify any advantages of the microemulsion as an efficient carrier to enhance the solubility of triterpene and adlay seed oil, and validate a definitely strong antitumor potency in $\mathrm{C} 57 \mathrm{~L} / 6 \mathrm{~J}$ mice bearing Lewis xenografts. The preliminary immunologic effects of treatment with these TMEs were also investigated.

\section{Materials and methods}

G. lucidum triterpene and adlay seed oil were prepared using supercritical $\mathrm{CO}_{2}$ extraction technology (purity $>62 \%$ and $75 \%$, respectively, as determined by ultraviolet spectroscopy). ${ }^{25,26}$ Labrafil ${ }^{\circledR}$ M 1944CS was received as a gift from Gattefossé Co, Ltd (Saint Priest Cedex, France). Cremophor ${ }^{\circledR}$ EL and Cremophor RH40 were generous gifts from BASF Co, Ltd (Ludwigshafen, Germany). Tween-80, Tween-60, Span-20, Tween-20, clove oil, castor oil, almond oil, and olive oil were provided by Sinopharma Group Co, Ltd (Shanghai, People's Republic of China). Polyethylene glycol (PEG)400, linoleic acid, isopropanol, propylene glycol, and isopropyl palmitate were purchased from Sigma-Aldrich St Louis, MO, USA. Water in this study was provided by the Milli-Q water purification system (Millipore Corporation, Billerica, MA, USA). All other chemicals and solvents were of analytical grade.

\section{Solubility studies}

To screen for a suitable oil, surfactant, and cosurfactant for preparation of the TMEs, the solubility of triterpene in various pharmaceutical adjuvants was evaluated using the saturated solubility method. Briefly, an excess amount of triterpene $(100 \mathrm{mg})$ was dissolved in $1 \mathrm{~mL}$ of various oils, including linoleic acid, oleic acid, triacetin, isopropyl palmitate, olive oil, adlay seed oil, clove oil, Labrafil M 1944CS, castor oil, and almond oil. Similarly, Cremophor RH40, Cremophor EL, Tween-80, Span-20, Tween-60, and Tween-20 were selected as candidates for the surfactant, and PEG400, ethanol, isopropanol, and propylene glycol were used to screen for the optimal cosurfactant. Each sample was stirred for 24 hours at room temperature, and then centrifuged at 11,000 rpm for 15 minutes. The concentration of triterpene in the obtained supernatant was detected by ultraviolet spectroscopy (UV-2800, Shanghai Aoxin Technology Instrument Co., Ltd., Shanghai, People's Republic of China).

\section{Construction of pseudoternary phase diagrams}

The pseudoternary phase diagram containing oil-surfactant/ cosurfactant-water was constructed using the water titration method to explore the optimal ratio between the various 
components as follows. First, the Cremophor RH40 as the surfactant was mixed with PEG400 as the cosurfactant $\left(\mathrm{S}_{\text {mix }}\right)$ in mass ratios $\left(\mathrm{K}_{\mathrm{m}}\right)$ of $1: 1,2: 1$, and $3: 1$. Next, oil phase (adlay seed oil or Labrafil M 1944CS and adlay seed oil $[2 / 1, \mathrm{w} / \mathrm{w}]$ ) and the $\mathrm{S}_{\text {mix }}$ were mixed further in mass ratios of $1: 9,2: 8,3: 7,4: 6,5: 5,6: 4,7: 3,8: 2$, and 9:1. Finally, deionized water was added dropwise into each mixture with gentle stirring to allow equilibration at $25^{\circ} \mathrm{C}$. The microemulsion phase was recognized as the region in the phase diagram where a translucent, easily flowable, and clear system was observed. The first axis of the pseudoternary phase diagram represented aqueous phase, the second axis corresponded to oil phase, and the third axis represented a combination of surfactant and cosurfactant at a prearranged ratio.

\section{Formulation and preparation of TMEs}

On the basis of the above findings, adlay seed oil (or adlay seed oil mixed with Labrafil M 1944CS, 1/2, w/w), Cremophor RH40, and PEG400 were used as the optimal oil, surfactant, and cosurfactant, respectively, for preparation of two TMEs with different mass ratios of triterpene to adlay seed oil (TME-1 and TME-2) using a conventional method as follows. ${ }^{27}$ Briefly, for TME-1 (triterpene/adlay seed oil, 1/1, w/w of crude drug), $75 \mathrm{mg}$ of triterpene was dissolved in a mixture of $132.5 \mathrm{mg}$ of adlay seed oil and $267.5 \mathrm{mg}$ of Labrafil M 1944CS, with vigorous magnetic stirring at room temperature for 12 hours. Next, $400 \mathrm{mg}$ of Cremophor RH40 and $200 \mathrm{mg}$ of PEG400 were added to the mixture, again with vigorous magnetic stirring at room temperature for a further 12 hours. At the end of this time, $2.6 \mathrm{~mL}$ of deionized water was added to the resulting mixture drop by drop. For TME-2 (triterpene/adlay seed oil, 1/3, w/w of crude drug), $75 \mathrm{mg}$ of triterpene and $400 \mathrm{mg}$ of adlay seed oil was mixed by vigorous magnetic stirring at room temperature for 12 hours and the subsequent procedures were similar to those used for TME-1.

\section{Characterization of TMEs}

Morphologic investigation of the TME formulations was done using transmission electron microscopy (TEM, JEOL Ltd, Tokyo, Japan). The sample was prepared by depositing a drop of diluted microemulsion solution onto a film-coated copper grid, staining with a drop of $2 \%$ aqueous solution of phosphotungstic acid prior to the experiment, and allowing it to dry at room temperature. The size and zeta potential of the TMEs were measured using a zeta potential analyzer
(Zeta Plus, Brookhaven, Holtsville, NY, USA). All measurements were carried out in triplicate.

\section{Content determination}

In this experiment, the concentration of triterpene was detected at $584 \mathrm{~nm}$ by ultraviolet spectrophotometry using ursolic acid as the reference substance, and the content of adlay seed oil was measured at $412 \mathrm{~nm}$ by ultraviolet spectrophotometry using glyceryl trioleate as the reference substance. ${ }^{28,29}$ Briefly, a calibration curve for ursolic acid was constructed using 5\% vanillic aldehyde-acetic acid and perchloric acid as a chromogenic agent at $60^{\circ} \mathrm{C}$, allowing calculation of the approximate triterpene content. Meanwhile, a calibration curve for glyceryl trioleate was constructed using a mixed chromogenic agent, comprising $0.1 \mathrm{~mol} / \mathrm{L} \mathrm{KCl}$ as a chloridion source, $2 \mathrm{~mol} / \mathrm{L}$ acetic acid and $0.05 \mathrm{~mol} / \mathrm{L}$ sodium periodate as an oxidant, and $0.5 \mathrm{~mL}$ of acetylacetone, $30 \mathrm{~mL}$ of isopropanol, $100 \mathrm{~mL}$ of ammonium acetate solution $(2 \mathrm{~mol} / \mathrm{mL})$, and $60 \mathrm{~mL}$ of acetic acid aqueous solution $(2 \mathrm{~mol} / \mathrm{mL})$ to calculate the approximate content of adlay seed oil.

\section{Evaluation of TMEs}

The adlay seed oil content in the microemulsion was determined as follows. Briefly, $1 \mathrm{~mL}$ of the TME was added to $9 \mathrm{~mL}$ of deionized water with ultrasonic extraction for 30 minutes. At the end of this pretreatment, the solution was filtered using a $0.45 \mu \mathrm{m}$ cellulose nitrate membrane and diluted 10 -fold with deionized water. Ultraviolet spectroscopy was then used to measure the content of triterpene and adlay seed oil. Finally, the resulting microemulsion was freeze-dried to measure the drug-loading rate.

Drug encapsulation efficiency (DEE) was calculated as follows: $\mathrm{DEE}=C_{1} / C_{2} \times 100 \%$, where $C_{1}$ represents the measured amount of triterpene or adlay seed oil in the TME after filtration, and $C_{2}$ is the total amount of triterpene or adlay seed oil in the TME. The drug-loading rate (DLE) was calculated as follows: DLE $=C_{3} / C_{4} \times 100 \%$, where $C_{3}$ represents the total measured amount of triterpene and adlay seed oil in the freeze-dried TME, and $C_{4}$ is the weight of the freeze-dried TME.

\section{Stability test}

In order to evaluate the stability of the TMEs in vitro, various critical factors including dilution, centrifugation, temperature, $\mathrm{pH}$ value, and ionic strength were investigated. The TMEs were diluted 10-fold and 100-fold with deionized water and saline, centrifuged at 13,000 rpm 
for 30 minutes, stored at $4^{\circ} \mathrm{C}$ and $24^{\circ} \mathrm{C}$ for 30 days, and dissolved at various $\mathrm{pH}$ levels to observe any morphologic changes.

\section{In vitro cytotoxicity}

\section{Cell culture}

A human lung cancer (A549) cell line and a murine lung cancer (Lewis) cell line were supplied by the Cell Bank at the Chinese Academy of Sciences. The cells were cultured in Roswell Park Memorial Institute-1640 medium with $10 \%(\mathrm{v} / \mathrm{v})$ fetal bovine serum, $100 \mathrm{U} / \mathrm{mL}$ penicillin, and $100 \mu \mathrm{g} / \mathrm{mL}$ streptomycin in an incubator at $37^{\circ} \mathrm{C}$ under an atmosphere of $5 \% \mathrm{CO}_{2}$ and $90 \%$ relative humidity. The cells were passaged by trypsin at a split ratio of $1: 8$ every 4-5 days (at $80 \%$ confluence).

\section{Preparation of test solutions}

In order to evaluate the ability of the TMEs to inhibit tumor growth, suspensions containing the same amount of G. lucidum triterpene and adlay seed oil (suspension-1 and suspension-2, 1/1 and 1/3 w/w of crude drug) corresponding to the TMEs using $0.2 \% \mathrm{CMC}-\mathrm{Na}$ as a deflocculant were prepared as positive control groups.

\section{Cytotoxicity studies}

To evaluate their potential toxicity to tumor cells, the antiproliferative effects of the TMEs and their corresponding suspensions against A549 cells and Lewis cells were assessed using the 3-(4,5-dimethylthiazol-2-yl)-2,5-diphenyltetrazolium bromide (MTT) colorimetric method. The A549 and Lewis cells were first seeded at a density of $5 \times 10^{4}$ cells per well in 96-well Costar ${ }^{\circledR}$ plates (Corning Life Sciences, Tewksbury, MA, USA) to reach $60 \%$ confluence. Afterwards, the culture medium was removed, and $200 \mu \mathrm{L}$ of the test solutions and a negative control $(200 \mu \mathrm{L}$ of fetal bovine serum-free culture medium) were incubated with the cells at $37^{\circ} \mathrm{C}$ for 48 hours. Next, $20 \mu \mathrm{L}$ of $5 \mathrm{mg} / \mathrm{mL}$ MTT in phosphate-buffered solution was added to each well for 4 hours at $37^{\circ} \mathrm{C}$ to stain the cells. Subsequently, the medium was removed, and the cells were mixed with $150 \mu \mathrm{L}$ of dimethyl sulfoxide. The absorbance was measured at $570 \mathrm{~nm}$ using an enzyme-linked immunosorbent assay (Thermo Scientific, Waltham, MA, USA). The relative cell inhibition rate (R\%) was calculated as follows:

$$
\mathrm{R} \%=1-\frac{\text { Absorbance }_{\text {test }}}{\text { Absorbance }_{\text {control }}} \times 100 \%
$$

\section{In vivo pharmacodynamics}

\section{Cell culture}

The method used to culture the Lewis cells was the same as that described for the in vitro study.

\section{Animals}

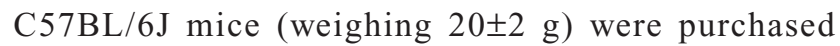
from the Silaike Laboratory Animal Co, Ltd (Shanghai, People's Republic of China). The mice were housed in polypropylene cages under standard laboratory conditions at a temperature of $25^{\circ} \mathrm{C} \pm 1^{\circ} \mathrm{C}$ and a relative humidity of $55 \% \pm 5 \%$. All animals used in this experiment were pathogen-free and allowed free access to food and water. The animal experiments were carried out in accordance with the protocol approved by the animal ethics committee at our institution.

\section{In vivo evaluation}

The ability of the TMEs and corresponding suspensions to inhibit tumor growth and improve immunity was evaluated to determine the synergistic effect of adlay seed oil and triterpene. Cyclophosphamide was used as a control because its high antitumor potency and immunosuppressive properties. C57BL/6 J mice bearing Lewis xenografts were generated by subcutaneous injection of Lewis cells $\left(2 \times 10^{6}\right.$ per mouse) into the right axilla. The mice were randomly divided into six groups $(n=6)$ on day 2 post-xenograft implantation, and treated with saline, cyclophosphamide $20 \mathrm{mg} / \mathrm{kg}$ per day, TMEs, or suspensions (high-dose, $30 \mathrm{~g}$ total crude drug/kg/day; low-dose, $15 \mathrm{~g}$ total crude drug/kg/day) by intragastric administration once daily for 14 days. Tumor growth was monitored by measuring the perpendicular diameter of the tumor with calipers. The estimated volume was calculated according to the formula: tumor volume $\left(\mathrm{mm}^{3}\right)=0.5 \times$ length $\times$ width $^{2}$. Preliminary immunologic effects were also investigated by weighing various tissues known to have an immune function, including the liver, thymus, and spleen. Body weight was recorded in each group as an indirect indicator of general toxicity. Plasma tumor necrosis factor-alpha and interleukin- 6 levels were detected by enzyme-linked immunosorbent assay (ELISA) kit at $37^{\circ} \mathrm{C}$.

\section{Statistical analysis}

The data are shown as the mean \pm standard deviation. Statistical significance was tested by the two-tailed Student's $t$-test, and was set at $P<0.05$. 


\section{Results and discussion \\ Screening of TME composition}

A drug with poor solubility might show unfavorable absorption in the gastrointestinal tract due to probable precipitation and low permeability. ${ }^{30-32}$ Solubilization of a microemulsion using a mixture of surfactants and oil to encapsulate insoluble drugs offers a means of preventing drug precipitation. Therefore, the solubility of triterpene in various pharmaceutical adjuvants was investigated to develop an optimal formulation.

The solubility of triterpene in oil phase is a critical factor for the drug-loading capacity of a microemulsion. Achieving higher drug solubility in oil phase requires lowering of the $\mathrm{S}_{\text {mix }}$ to maintain the miscibility of the oils, thereby reducing unwanted effects in normal tissues. As shown in Table 1, triterpene had the highest solubility at $78.36 \pm 3.65 \mathrm{mg} / \mathrm{mL}$ and $66.61 \pm 1.27 \mathrm{mg} / \mathrm{mL}$ in adlay seed oil and Labrafil $\mathrm{M}$ 1944CS, respectively. Importantly, as an active ingredient in the Li-Ying formulation, adlay seed oil would act as a functional oil phase in the construction of a novel triterpeneloaded microemulsion, and would be expected to increase the drug-loading rate and reduce the cytotoxicity of the bare microemulsion carrier. In the present work, in order to

Table I Solubility of triterpene in various oils, surfactants, and cosurfactants at $25^{\circ} \mathrm{C}$

\begin{tabular}{lll}
\hline Component & Solvant & $\begin{array}{l}\text { Concentration } \\
(\mathbf{m g} / \mathbf{m L})\end{array}$ \\
\hline Oils & Linoleic acid & $51.83 \pm 3.69$ \\
& Oleic acid* & $31.18 \pm 1.94$ \\
& Triacetin & $29.37 \pm 5.14$ \\
& Isopropyl palmitate & $31.62 \pm 2.96$ \\
& Olive oil* & $22.18 \pm 2.24$ \\
& Adlay seed oil & $78.36 \pm 3.65$ \\
& Clove oil & $12.38 \pm 2.53$ \\
& Labrafi ${ }^{\circledR}$ M 1944CS* & $66.61 \pm 1.27$ \\
Surfactants & Castor oil & $17.31 \pm 2.54$ \\
& Almond oil & $36.51 \pm 1.49$ \\
& Cremophor ${ }^{\circledR}$ EL & $27.65 \pm 1.82$ \\
& Tween-80 & $26.17 \pm 3.17$ \\
& Tween-60 & $21.43 \pm 1.99$ \\
& Span-20 & $16.51 \pm 1.24$ \\
& Cremophor RH40* & $37.89 \pm 2.20$ \\
Cosurfactants* & Tween-20* & $40.37 \pm 1.16$ \\
& PEG400 & $33.73 \pm 3.51$ \\
& Ethanol & $45.12 \pm 3.63$ \\
& Isopropanol & $31.27 \pm 3.13$ \\
& Propylene glycol & $21.43 \pm 1.88$ \\
\hline
\end{tabular}

Notes: *Published data; each value represents the mean \pm standard deviation $(n=3)$. Abbreviation: PEG, polyethylene glycol. maintain the prearranged ratio of triterpene to adlay seed oil and to prepare the microemulsion successfully, Labrafil M 1944CS was also mixed with adlay seed oil as a mixed oil phase in the preparation of TME-1.

Due to their nontoxicity, biocompatibility, and stability at various $\mathrm{pH}$ values, nonionic surfactants such as Cremophor RH40, Tween-20, Tween-60, Tween-80, Span-20, and Cremophor EL were chosen as candidates when screening for the optimal surfactant. The solubility of triterpene in PEG400, ethanol, isopropanol, and propylene glycol was also measured. Cremophor RH40 and Tween-20 showed higher solubilization for triterpene in comparison with the other surfactants. In view of the poor microemulsification capacity and probable hemolytic behavior of Tween-20, Cremophor RH40 was used as the optimal surfactant. Further, PEG400 was used as a cosurfactant because of its acceptable solubility $(33.73 \pm 3.51 \mathrm{mg} / \mathrm{mL})$ and nonimmunogenicity.

\section{Construction of phase diagrams}

Pseudoternary phase diagrams are widely used for determination of the zone of microemulsion formation. According to the above-selected composition, phase diagrams were constructed using adlay seed oil as the oil phase, Cremophor RH40 as the surfactant, and PEG400 as the cosurfactant. Pseudoternary phase diagrams were constructed to identify the microemulsion region by optimizing the content of $\mathrm{S}_{\text {mix }}$, oil, and water. As shown in Figure 1A, the total area of the microemulsion increased noticeably compared with those using other ratios when $\mathrm{K}_{\mathrm{m}}$ was 2:1. In order to maintain the ratio of triterpene to adlay seed oil for further in vivo evaluation, another TME using Labrafil M 1944CS and adlay seed oil as the mixed oil phase was prepared. A similar phase diagram was observed (see Figure 2B).

\section{Preparation of TMEs}

TME-2 using adlay seed oil as oil phase containing triterpene and adlay seed oil (1/3, w/w of crude drug) was prepared by the conventional drop method at room temperature. To clarify the superiority of such a drug delivery system with regard to antitumor activity and explore the optimal mass ratio of triterpene and adlay seed oil, TME-1 containing triterpene and adlay seed oil at a weight ratio of 1:1 (crude drug) was also synthesized. In order to offset the effect of the amount of oil phase and prepare the microemulsion successfully, Labrafil M 1944CS was mixed with adlay seed oil to maintain the ratio of oil to $S_{\text {mix }}$ at 2:3. 
A
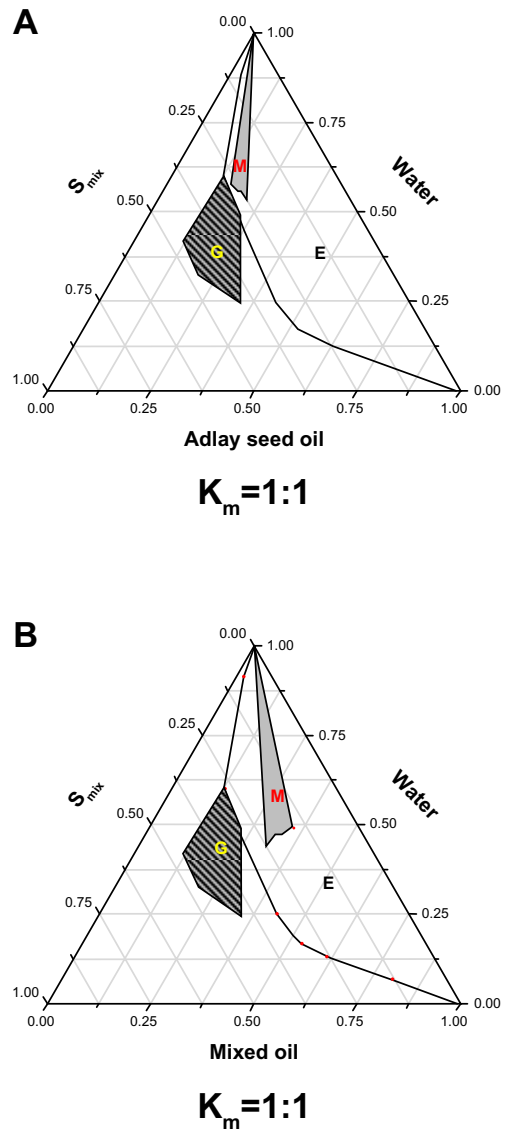

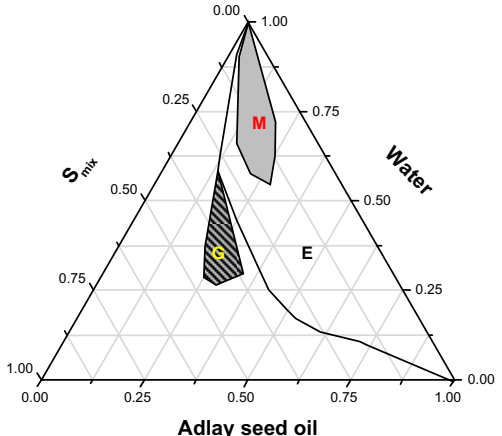

$\mathrm{K}_{\mathrm{m}}=2: 1$

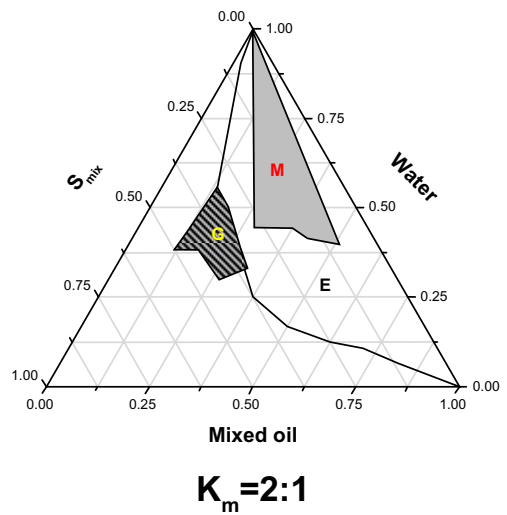

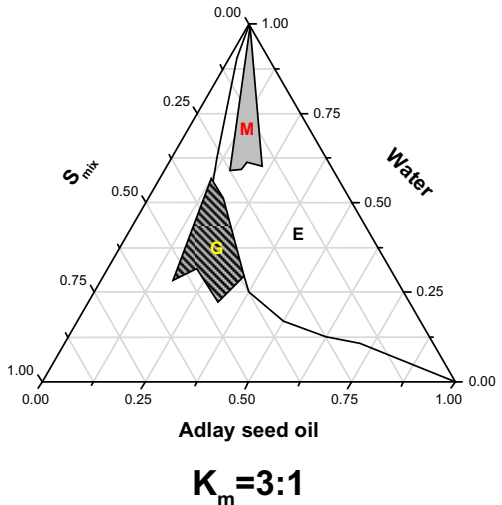

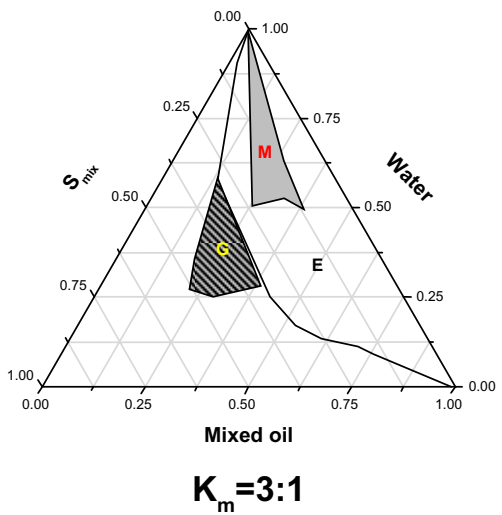

Figure I Pseudoternary phase diagrams of microemulsion composed of (A) adlay seed oil and (B) mixed oil (Labrafil ${ }^{\circledR}$ [Gattefossé Co, Ltd, Saint Priest Cedex, France] M I944CS-adlay seed oil, 2/I, w/w), Cremophor ${ }^{\circledR}$ (BASF Co, Ltd, Ludwigshafen, Germany) RH40, water, and PEG400 at different K $\mathrm{m}_{\mathrm{m}}$ values. G, M, and E represent the zones of gel, microemulsion, and emulsion, respectively.

\section{Characteristics of TMEs}

The size and zeta potential of the TMEs were analyzed with dynamic light scattering, and morphologic investigation was done by TEM. As shown in Table 2, the particle size of TME-1 and TME- 2 was $41.13 \pm 4.11 \mathrm{~nm}$ and $47.59 \pm 5.55 \mathrm{~nm}$, respectively, with a narrow size distribution. The zeta potential of the TME-1 and TME- 2 was $-13.74 \pm 2.75 \mathrm{mV}$ and $-16.98 \pm 1.48 \mathrm{mV}$, respectively, with no significant difference in size or zeta potential after incorporation of Labrafil M 1944CS as an auxiliary oil phase. Further, the TEM images
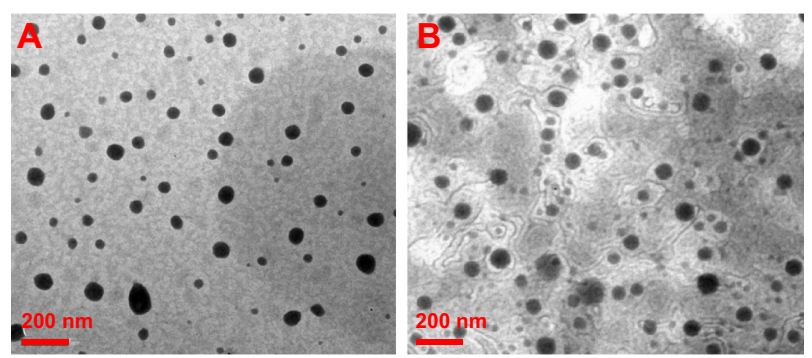

Figure 2 Morphologic patterns. Transmission electron microscopic images of (A) TME-I and (B) TME-2 at room temperature.

Abbreviation: TME, triterpene-loaded microemulsion. in Figure 2 indicate that the TMEs were nearly spherical with a small size and narrow distribution, suggesting that the microemulsions were dispersed homogeneously in aqueous medium. These results are consistent with the data from dynamic light scattering.

\section{Content determination}

Due to their similar chemical structures, ursolic acid and glyceryl trioleate were used as reference substances for determination of the triterpene and adlay seed oil content, respectively. Being a type of triterpene, ursolic acid can be colored by a "vanillic aldehyde-perchloric acid" chromogenic reaction and its quantity determined by ultraviolet spectroscopy with a good correlation. The total content of G. lucidum triterpene was calculated from the calibration curves for ursolic acid using the above chromogenic method. Triglyceride was the main component of adlay seed oil, and could be detected using a specific chromogenic reaction. In this study, the calibration curve for glyceryl trioleate was constructed to calculate the total triglyceride content in adlay seed oil. According to the above method, the loading efficiency of 
Table 2 Characteristics of triterpene-loaded microemulsions

\begin{tabular}{lllllll}
\hline Formulation & Size $(\mathbf{n m})$ & PDI & Zeta $(\mathbf{m V})$ & DEE $(\%)^{\mathrm{a}}$ & ${\text { DEE }(\%)^{\mathrm{b}}}^{\text {DLE }(\%)}$ \\
\hline TME-I & $41.13 \pm 4.1 \mathrm{I}$ & $0.199 \pm 0.034$ & $-13.74 \pm 2.75$ & $83.92 \pm 1.82$ & $84.27 \pm 3.21$ & $4.18 \pm 0.57$ \\
TME-2 & $47.59 \pm 5.55$ & $0.156 \pm 0.045$ & $-16.98 \pm 1.48$ & $79.32 \pm 2.19$ & $81.76 \pm 1.65$ & $9.87 \pm 0.29$ \\
\hline
\end{tabular}

Notes: DEE (\%) and DEE (\%) ${ }^{\mathrm{b}}$ represent the DEE of adlay seed oil and triterpene, respectively; each value represents the mean \pm standard deviation $(\mathrm{n}=3)$.

Abbreviations: PDI, polydispersity index; DEE, drug encapsulation efficiency; DLE, drug-loading rate; TME, triterpene-loaded microemulsion.

TME- 1 and TME- 2 were calculated to be $4.18 \% \pm 0.57 \%$ and $9.87 \% \pm 0.29 \%$, respectively. TME- 2 using adlay seed oil as oil phase brought about a significantly higher loading rate compared with TME-1 using adlay seed oil and Labrafil M 1944CS as mixed oil phase. Further, the encapsulation efficiency of adlay seed oil and triterpene in TME-1 was $83.92 \% \pm 1.82 \%$ and $84.27 \% \pm 3.21 \%$, and in TME- 2 was $79.32 \% \pm 2.19 \%$ and $81.76 \% \pm 1.65 \%$, respectively. The encapsulation efficiency of triterpene and adlay seed oil was not decreased noticeably when the drug-loading rate was significantly enhanced, suggesting a satisfactory preparation technology.

\section{Stability studies}

Understanding the stability of a microemulsion under various environments, including centrifugation, temperature, $\mathrm{pH}$, and dilution with deionized water or saline, is important to determine its structural integrity and fate in vivo. No obvious change in size of a TME would be considered an indication of stability. In this study, no noticeable creaming was observed after centrifugation at $13,000 \mathrm{rpm}$ for 30 minutes, and no turbidity or flocculation was evident after storage at $4^{\circ} \mathrm{C}$ or $24^{\circ} \mathrm{C}$ for 30 days. Importantly, as shown in Figure 3A, the size of the TMEs changed very little with a low polydispersity index when the TME formulations were diluted 10-fold and 100-fold with deionized water and saline, demonstrating potential dilution stability. The $\mathrm{pH}$ tolerance was evaluated for the TMEs at a $\mathrm{pH}$ range of 2.0-8.0, and there was little change in size and a low polydispersity index was maintained (see Figure 3B), indicating acceptable stability in the gastrointestinal tract and hence enhancement on absorption provided by the nanosized structure. Therefore, the microemulsion we prepared may be a potential drug delivery system for enhancement of antitumor efficacy by virtue of a stable structure.

\section{Antiproliferative effects in vitro}

The antiproliferative effects of the TMEs against A549 cells and Lewis cells in vitro were evaluated using the MTT assay. Suspensions containing the same amount of triterpene and adlay seed oil as the corresponding TMEs using ethanol and phosphate buffer salt as the component solvent were used as a positive control to confirm the marked effect of TMEs in terms of suppressing tumor cell proliferation. As shown in Figure 4A, dose-dependent antiproliferative activity was found for both the TME and suspension formulations, and the TMEs showed an enhanced effect relative to that of the suspension formulations at the same concentration. The halfmaximal inhibitory concentrations of TME-1 and TME-2 against A549 cells were calculated to be $0.77 \mathrm{mg}$ crude $\mathrm{drug} / \mathrm{mL}$ and $0.62 \mathrm{mg}$ crude $\mathrm{drug} / \mathrm{mL}$, respectively, and were 1.84-fold and 2.5-fold improved relative to that of the
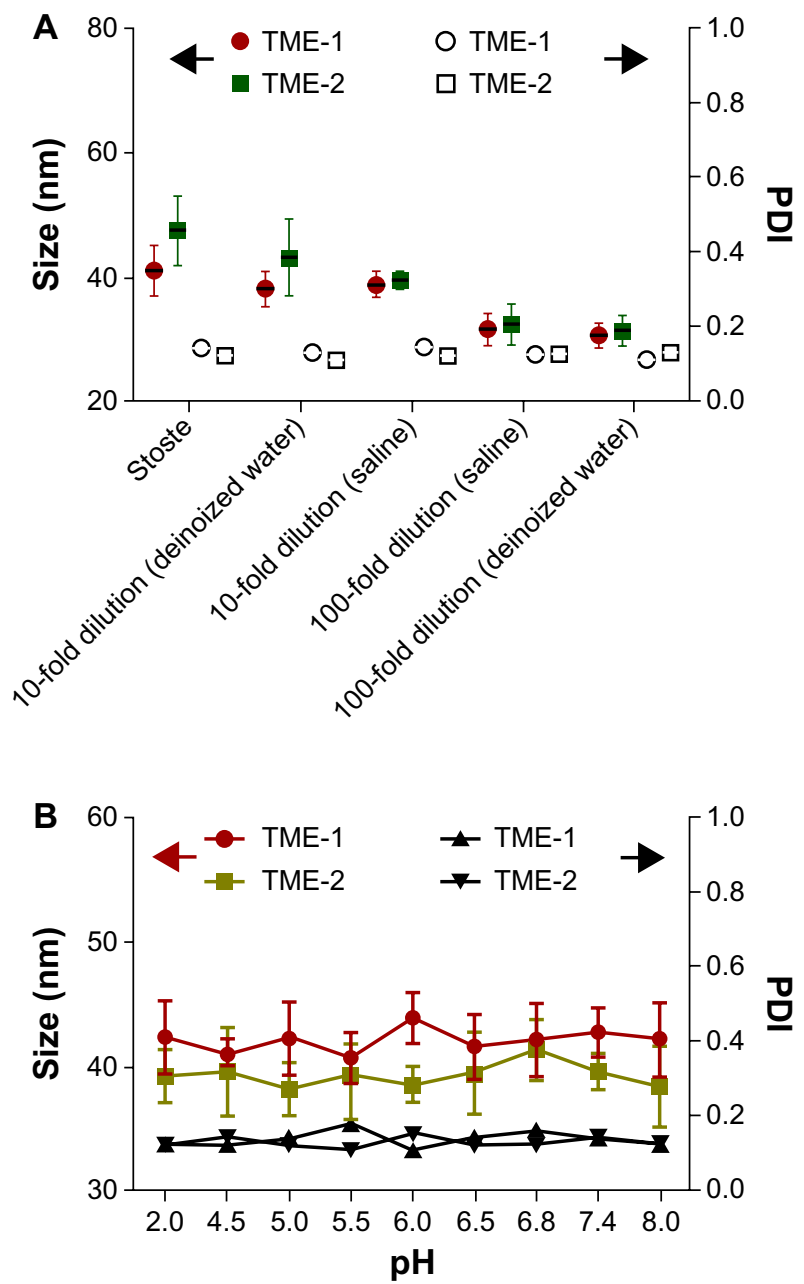

Figure 3 Stability of TMEs after (A) dilution with deionized water and saline and (B) stimulation at various $\mathrm{pH}$ values $(\mathrm{n}=6)$.

Abbreviations: PDI, polydispersity index; TME, triterpene-loaded microemulsion. 
corresponding suspension formulations. Similar cytotoxicity results were seen in the Lewis cell line. In addition, the bare pharmaceutical adjuvant in the microemulsions showed no cytotoxicity against A549 cells or Lewis cells under the same conditions (see Figure 4C and D). Interestingly, due to introduction of adlay seed oil as oil phase, the bare ME-2 containing less pharmaceutical adjuvant showed less cytotoxicity in both the cell lines, suggesting potential safety in vivo.

\section{Antitumor efficacy in vivo}

To estimate the feasibility of using TMEs for cancer therapy in vivo, their antitumor efficacy and their effects on tissue and body weight were studied in a xenograft model of murine Lewis lung carcinoma. ${ }^{33,34}$ As shown in Figure 5A, TME-2 at a dose of $30 \mathrm{mg}$ crude $\mathrm{drug} / \mathrm{kg} /$ day had a remarkable inhibitory effect on tumor size in comparison with the

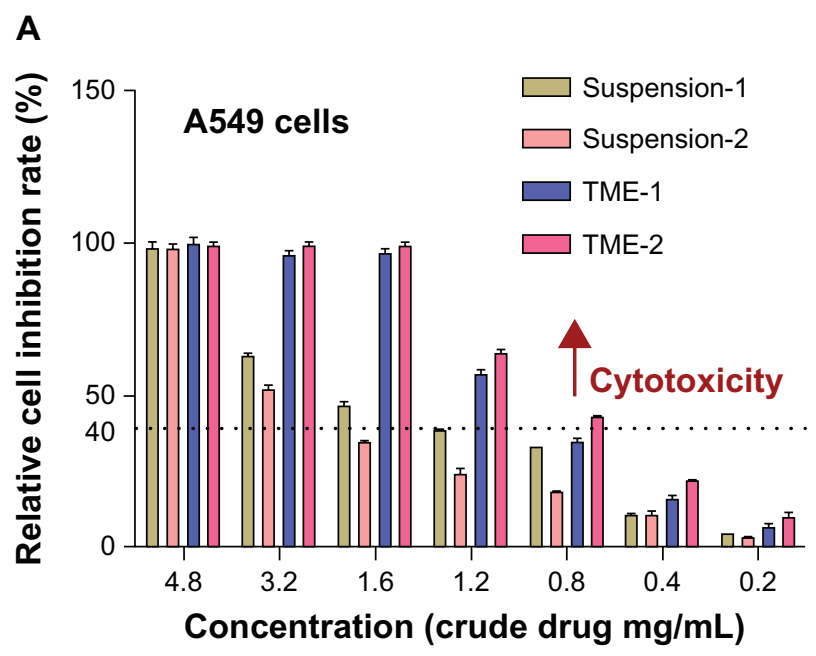

B

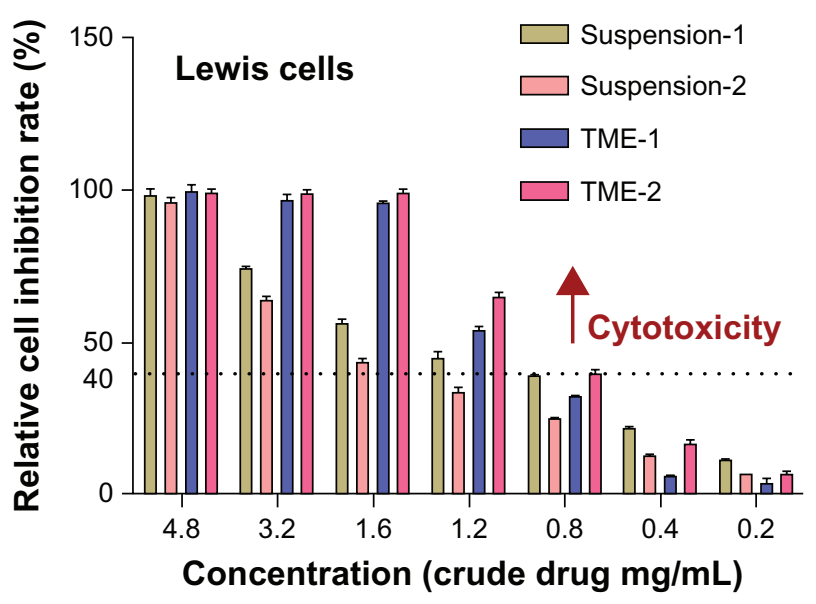

other formulations, including saline $(P<0.05)$ and the TME-1 formulation $(P<0.05)$, indicating significantly improved intestinal absorption and increased bioavailability. Further, no noticeable difference in inhibition of tumor growth between cyclophosphamide and TME-2 (high-dose) was found, indicating good antitumor efficacy and promising prospects for clinic application. In addition, no observable difference in body weight was observed between the saline and triterpene-adlay seed oil formulations, indicating low toxicity in vivo compared with cyclophosphamide (Figure 5B). To assess the toxicity of the various formulations in susceptible tissues, the weight to body ratio of the liver, thymus, and spleen was evaluated. The results shown in Figure 6 demonstrate that suspension-1 and TME-2 at high-dose caused a significant increase in the weight of the liver, thymus, and spleen compared with cyclophosphamide as an immunosuppressant, suggesting a superior effect
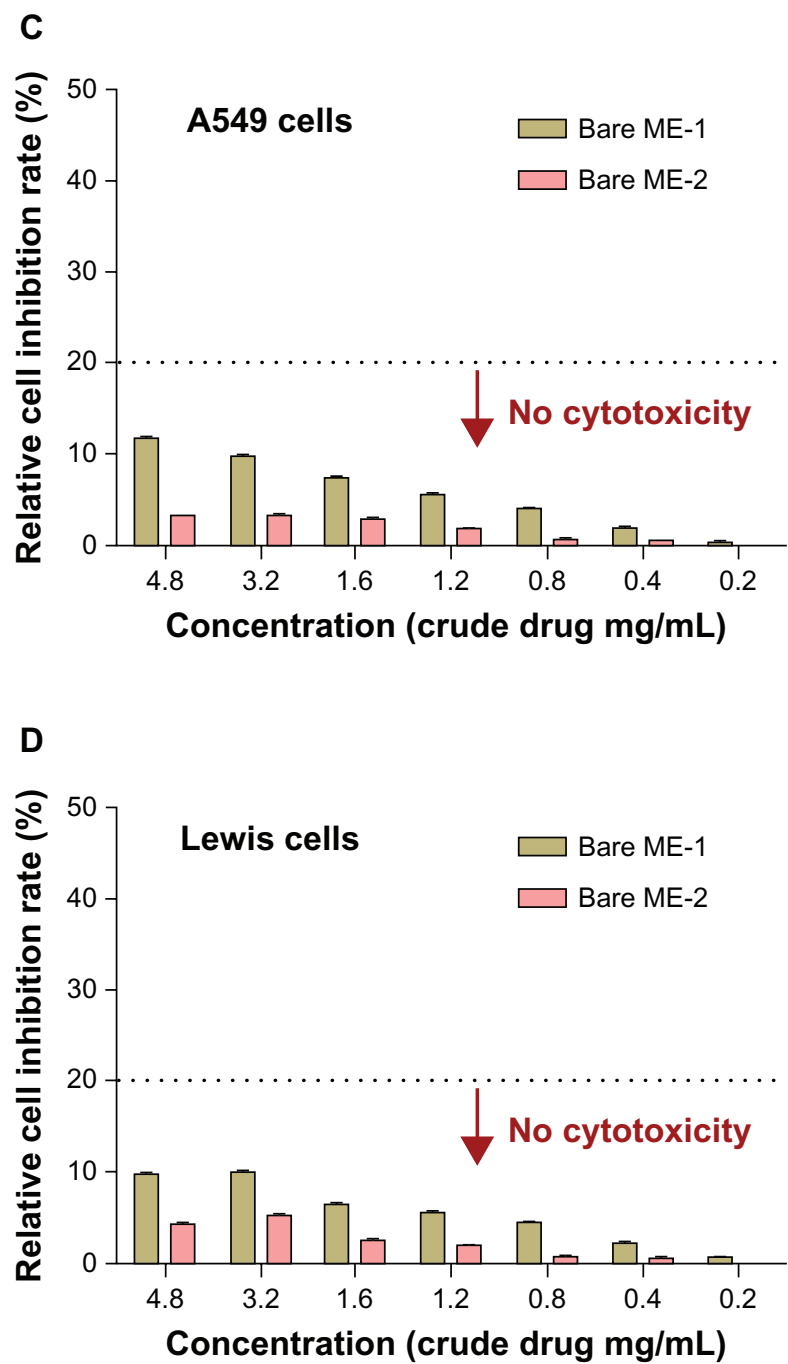

Figure 4 In vitro antiproliferative effects, showing cytotoxicity of suspension and microemulsion formulations against a (A) A549 cell line and a (B) Lewis cell line at various concentrations for 48 hours $(n=6)$. The dotted line indicates more than $40 \%$ inhibition, defined as cytotoxic. Cytotoxicity of bare ME-I (containing Labrafi ${ }^{\circledR}$ [Gattefossé Co, Ltd, Saint Priest Cedex, France] M 1944CS, Cremophor ${ }^{\circledast}$ [BASF Co, Ltd, Ludwigshafen, Germany] RH40 and PEG400) and bare ME-2 (containing Cremophor RH40 and PEG400) against a (C) A549 cell line and a (D) Lewis cell line at concentrations equivalent to those of the corresponding drug-loaded formulations for 48 hours ( $\mathrm{n}=6$ ). The dotted line indicates less than $20 \%$ inhibition, defined as noncytotoxic.

Abbreviations: ME, microemulsion; TME, triterpene-loaded microemulsion. 

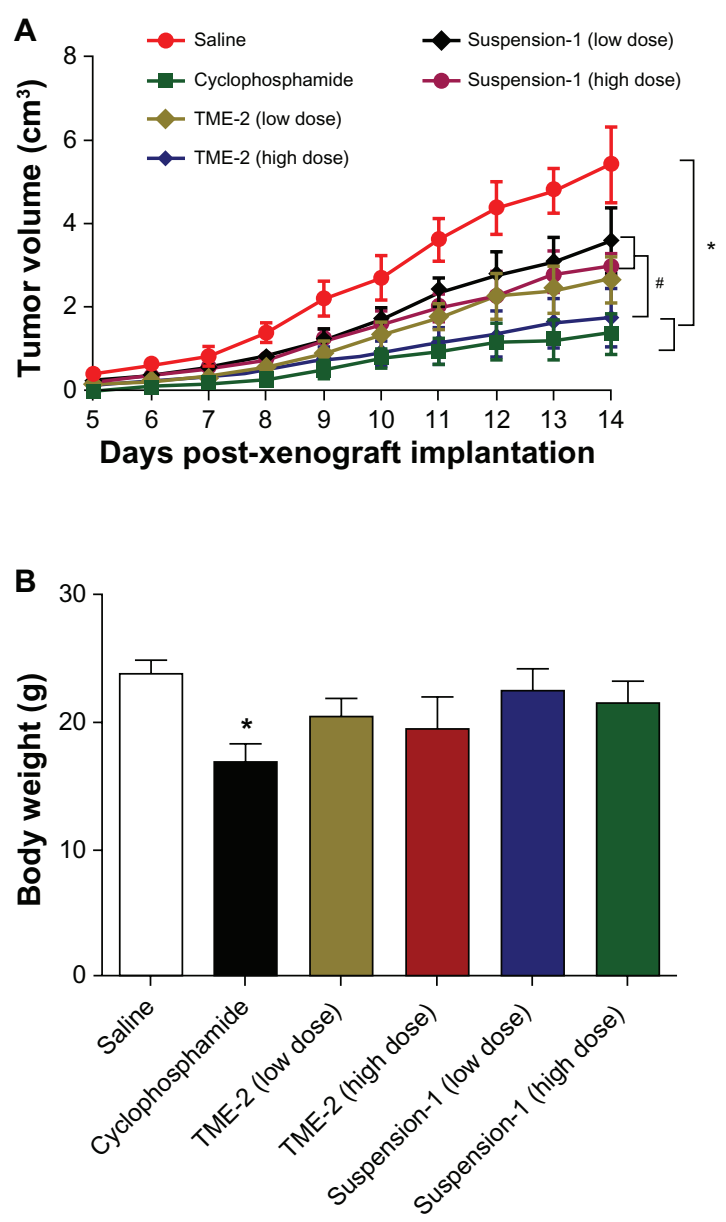

Figure 5 In vivo evaluation. (A) Antitumor efficacy against Lewis xenograft tumor after intragastric administration of various formulations of triterpene and adlay seed oil at a dose of $30 \mathrm{~g}$ crude drug $/ \mathrm{kg}$ and $15 \mathrm{~g}$ crude drug $/ \mathrm{kg}$. (B) Weight of mice in each group at day 14 after tumor implantation. $* P<0.05$ versus saline; ${ }^{\# P}<0.05$ versus TME-2 (high-dose), $\mathrm{n}=6$.

Abbreviation: TME, triterpene-loaded microemulsion.
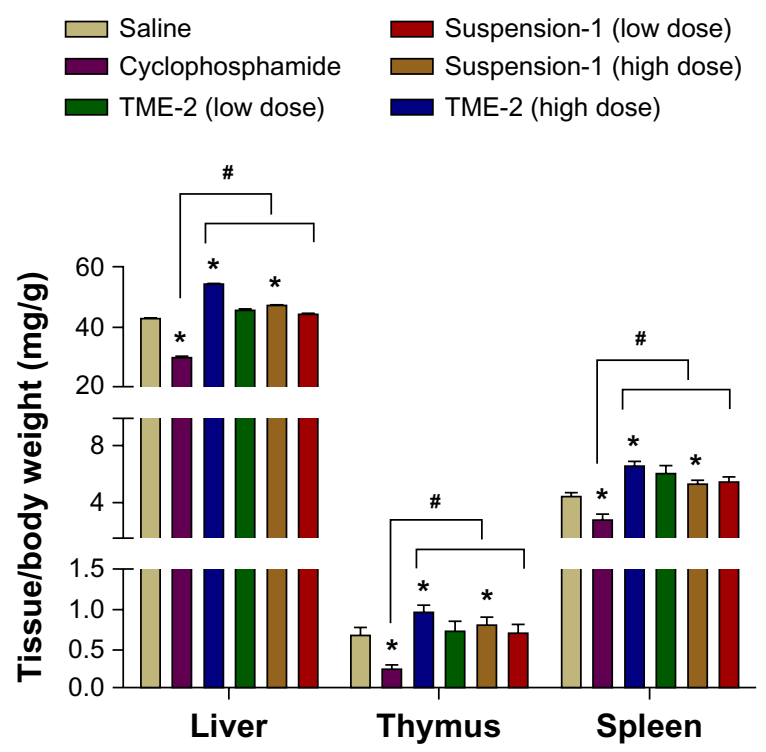

Figure 6 Relative weight of liver, thymus, and spleen after intragastric administration of the different formulations on day 14 post-xenograft implantation. ${ }^{*} P<0.05$ versus saline; ${ }^{P}<0.05$ versus cyclophosphamide $(n=6)$.

Abbreviation: TME, triterpene-loaded microemulsion.

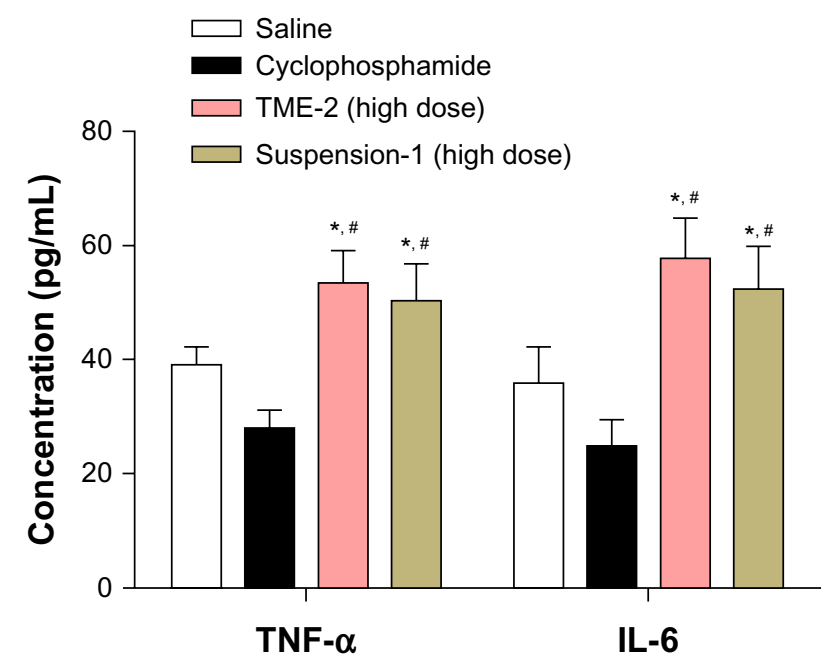

Figure 7 TNF- $\alpha$ and IL- 6 levels after intragastric administration of the different formulations for 14 days $(n=6)$ after tumor implantation. $* P<0.05$ versus saline; ${ }^{\#} \mathrm{P}<0.05$ versus cyclophosphamide.

Abbreviations: IL-6, interleukin-6; TNF- $\alpha$, tumor necrosis factor-alpha; TME, triterpene-loaded microemulsion.

on immunoregulation. Further, some immunologic indices, such as levels of tumor necrosis factor-alpha and interleukin-6, were also measured after intragastric administration for 14 days as a preliminary immunologic evaluation at the quantitative level. As shown in Figure 7, significantly higher tumor necrosis factor-alpha and IL-6 levels were observed in comparison with saline $(P<0.05)$ and cyclophosphamide $(P<0.05)$ after treatment with TME- 2 and suspension- 1 at high-dose, indicating that TME-2 improves immunity as well as having potent antitumor activity.

\section{Conclusion}

We developed a TME formulation using adlay seed oil as a functional oil phase for enhancement of the solubility of triterpene. The optimal preparation technology was determined at a ratio of 1:3:4 (triterpene/adlay seed oil $/ \mathrm{S}_{\text {mix }}$, $\mathrm{w} / \mathrm{w} / \mathrm{w}$ of crude drug). The characteristics of TMEs were investigated, and the drug-loading rate was found to be significantly increased when using adlay seed oil as oil phase. The structure of the TMEs was relatively stable in various storage conditions. In vitro, the TMEs had a stronger inhibitory effect on growth of A549 cells and Lewis cells in comparison with the suspension formulations. Importantly, our in vivo results suggest a promising nanosized vehicle for improving the solubility of poorly soluble drugs, increasing their absorption and therapeutic efficacy, and leading to more advanced development of cancer therapy.

\section{Acknowledgment}

This work was supported financially by the National Natural Science Foundation (81373979) and the Jiangsu 
Provincial Chinese Medicine Leading Talent Project (LJ200913).

\section{Disclosure}

The authors report no conflicts of interest in this work.

\section{References}

1. Jiao C, Xie YZ, Yang X, et al. Anticancer activity of Amauroderma rude. PLoS One. 2013;8:e66504.

2. Suarez-Arroyo IJ, Rosario-Acevedo R, Aguilar-PerezA, et al. Anti-tumor effects of Ganoderma lucidum (reishi) in inflammatory breast cancer in in vivo and in vitro models. PLoS One. 2013;8:e57431.

3. Pan K, Jiang Q, Liu G, Miao X, Zhong D. Optimization extraction of Ganoderma lucidum polysaccharides and its immunity and antioxidant activities. Int J Biol Macromol. 2013;55:301-306.

4. Yue GG, Chan BC, Han XQ, et al. Immunomodulatory activities of Ganoderma sinense polysaccharides in human immune cells. Nutr Cancer. 2013;65:765-774.

5. Yu YL, Lu Y, Tang X, Cui FD. Formulation, preparation and evaluation of an intravenous emulsion containing Brucea javanica oil and Coix seed oil for anti-tumor application. Biol Pharm Bull. 2008;31: 673-680.

6. Yu F, Gao J, Zeng Y, Liu CX. Inhibition of Coix seed extract on fatty acid synthesis, novel target for anticancer activity. J Ethnopharmacol. 2008;119:252-258.

7. Wang JL, Gu T, Zhong JJ. Enhanced recovery of antitumor ganoderic acid T from Ganoderma lucidum mycelia by novel chemical conversion strategy. Biotechnol Bioeng. 2012;109:754-762.

8. Jedinak A, Thygarajan-Sahu A, Jiang J, Sliva D. Ganodermanontriol, a lanostanoid triterpene from Ganoderma lucidum, suppresses growth of colon cancer cells through ß-catenin signaling. Int J Oncol. 2011;38:761-767

9. Thyagarajan A, Jedinak A, Nguyen H, et al. Triterpenes from Ganoderma lucidum induce autophagy in colon cancer through the inhibition of p38 mitogen-activated kinase (p38 MAPK). Nutr Cancer. 2010;62:630-640.

10. Lu J, Sun LX, Lin ZB, et al. Antagonism by Ganoderma lucidum polysaccharides against the suppression by culture supernatants of B16F10 melanoma cells on macrophage. Phytother Res. 2013;5: 1117-1122.

11. Nornoo AO, Zheng H, Lopes LB, Johnson-Restrepo B, Kannan K, Reed R. Oral microemulsions of paclitaxel: in situ and pharmacokinetic studies. Eur J Pharm Biopharm. 2009;71:310-317.

12. Davis T, Farag SS. Treating relapsed or refractory Philadelphia chromosome-negative acute lymphoblastic leukemia: liposomeencapsulated vincristine. Int J Nanomedicine. 2013;8:3479-3488.

13. Bhardwaj V, Ankola DD, Gupta SC, Schneider M, Lehr CM, Kumar MN. PLGA nanoparticles stabilized with cationic surfactant: safety studies and application in oral delivery of paclitaxel to treat chemicalinduced breast cancer in rat. Pharm Res. 2009;26:2495-2503.

14. Dabholkar RD, Sawant RM, Mongayt DA, Devarajan PV, Torchilin VP. Poly-ethylene glycol-phosphatidylethanolamine conjugate (PEG-PE)based mixed micelles: some properties, loading with paclitaxel, and modulation of P-glycoprotein-mediated efflux. Int J Pharm. 2006;315: $148-157$.

15. Yi C, Zhong H, Tong S, et al. Enhanced oral bioavailability of sterolloaded microemulsion formulation of Flammulina velutips, a potential antitumor drug. Int J Nanomedicine. 2012;7:5067-5078.

16. Lu Y, Wu K, Li L, et al. Characterization and evaluation of an oral microemulsion containing the antitumor ditrpenoid compound ent11alpha-hydroxy-15-oxo-kaur-16-en-19-oic-acid. Int J Nanomedicine. 2013;8:1879-1886.
17. Baek MK, Lee JH, Cho YH, Kim HH, Lee GW. Self-microemulsifying drug-delivery system for improved oral bioavailability of pranlukast hemihudrate: preparation and evaluation. Int J Nanomedicine. 2013;8:167-176.

18. Cho YD, Park YJ. In vitro and in vivo evaluation of a selfmicroemulsifying drug delivery system for the poorly soluble drug fenofibrate. Arch Pharm Res. June 11, 2013. [Epub ahead of print.]

19. Deshmukh A, Kulkarni S. Solid self-microemulsifying drug delivery system of ritonavir. Drug Dev Ind Pharm. March 7, 2013. [Epub ahead of print.]

20. Van Speybroeck M, Williams HD, Nguyen TH, Anby MU, Porter CJ, Augustijns P. Incomplete desorption of liquid excipients reduces the in vitro and in vivo performance of self-emulsifying drug delivery systems solidified by adsorption onto an inorganic mesoporous carrier. Mol Pharm. 2012;9:2750-2760.

21. Sahle FF, Wohlrab J, Neubert RH. Controlled penetration of ceramides into and across the stratum corneum using various types of microemulsions and formulation associated toxicity studies. Eur $J$ Pharm Biopharm. June 27, 2013. [Epub ahead of print.]

22. Akter N, Radiman S, Mohamed F, Reza MI. Self-assembled potential bionanocarriers for drug delivery. Mini Rev Med Chem. 2013;13: 1327-1339.

23. Han M, Fu S, Fang XL. [Screening of Panax notoginsenoside water in oil microemulsion formulations and their evaluation in vitro and in vivo]. Yao Xue Xиe Bao. 2007;42:780-786. Chinese.

24. Okonogi S, Chaiyana W. Enhancement of anti-cholinesterase activity of Zingiber cassumunar essential oil using a microemulsion technique. Drug Discov Ther. 2012;6:249-255.

25. Ge FH, Li Y, Xie JM, et al. [Studies on technology of supercritical$\mathrm{CO} 2$ fluid extraction for volatile oils and saikosaponins in Bupleurum chinense DC]. Zhongguo Zhong Yao Za Zhi. 2000;25:149-153. Chinese.

26. Chen GY, Shi ZH, Li HC, Ge FH, Zhan HS. [Supercritical CO2 extraction and component analysis of Aesculus wilsonii seed oil]. Zhong Yao Cai. 2013;36(3):475-478. Chinese.

27. Zheng HM, Li HB, Wang da W, Liu D. Preparation methods for monodispersed garlic oil microspheres in water using the microemulsion technique and their potential as antimicrobials. J Food Sci. 2013;78: N1301-N1306.

28. Baiocchi C, Medana C, Giancotti V, et al. Qualitative characterization of Desmodium adscendens constituents by high-performance liquid chromatography-diode array ultraviolet-electrospray ionization multistage mass spectrometry. Eur J Mass Spectrom (Chichester, Eng). 2013;19:1-15.

29. Yu F, Gao J, Zeng Y, Liu CX. Effects of adlay seed oil on blood lipids and antioxidant capacity in hyperlipidemic rats. J Sci Food Agric. 2011;91:1843-1848.

30. Mo R, Jin X, Li N, et al. The mechanism of enhancement on oral absorption of paclitaxel by $N$-octyl- $O$-sulfate chitosan micelles. Biomaterials. 2011;32:4609-4620.

31. Patel NR, Pattni BS, Abouzeid AH, Torchilin VP. Nanopreparations to overcome multidrug resistance in cancer. Adv Drug Deliv Rev. August 23, 2013. [Epub ahead of print.]

32. Sharma P, Garg S. Pure drug and polymer based nanotechnologies for the improved solubility, stability, bioavailability and targeting of antiHIV drugs. Adv Drug Deliv Rev. 2010;62:491-502.

33. Tian D, Sun Y, Yang Y, Lei M, Ding N, Han R. Human telomerase reverse-transcriptase promoter-controlled and herpes simplex virus thymidine kinase-armed adenoviruses for renal cell carcinoma treatment. Onco Targets Ther. 2013;6:419-426.

34. Chen CY, Jan CI, Lo JF, et al. Tid1-L inhibits EGFR signaling in lung adenocarcinoma by enhancing EGFR ubiquitinylation and degradation. Cancer Res. 2013;73:4009-4019. 
International Journal of Nanomedicine

Dovepress

\section{Publish your work in this journal}

The International Journal of Nanomedicine is an international, peerreviewed journal focusing on the application of nanotechnology in diagnostics, therapeutics, and drug delivery systems throughou the biomedical field. This journal is indexed on PubMed Central, MedLine, CAS, SciSearch ${ }^{\circledR}$, Current Contents ${ }^{\circledR} /$ Clinical Medicine,
Journal Citation Reports/Science Edition, EMBase, Scopus and the Elsevier Bibliographic databases. The manuscript management system is completely online and includes a very quick and fair peer-review system, which is all easy to use. Visit http://www.dovepress.com/ testimonials.php to read real quotes from published authors.

Submit your manuscript here: http://www.dovepress.com/international-journal-of-nanomedicine-journal 